\title{
Patterned Shape Memory Alloy Films
}

\author{
Kiyotaka Mori *, Jian Li, Alexander L. Roytburd and Manfred Wuttig \\ Department of Materials Science and Engineering, University of Maryland, College Park, \\ Maryland 20742-2115, USA
}

The uniaxial stress field in shape memory alloy (SMA) films patterned into thin strips increases the transformation induced deflection of SMA/Si cantilever bimorphs in comparison to cantilevers with planar films. In the single phase temperature ranges $T>A_{\mathrm{f}}, M_{\mathrm{S}}$ and $T<A_{\mathrm{s}}$, $M_{\mathrm{f}}\left(A_{\mathrm{f}}\right.$-austenite finish, $A_{\mathrm{s}}$-austenite start, $M_{\mathrm{f}}$-martensite finish and $M_{\mathrm{s}}$-martensite start temperatures), where the deflection is controlled by the thermoelastic stress, the change reflects the difference between the uniaxial and biaxial stress states. In the temperature regimes $A_{\mathrm{s}}<T<A_{\mathrm{f}}$, $M_{\mathrm{f}}<T<M_{\mathrm{s}}$, the martensitic microstructures created by uni- vs. biaxial stress fields are responsible for the difference of the cantilever deflection.

(Received November 12, 2001; Accepted April 17, 2002)

Keywords: martensite, films, stress relief, stress state, NiTi, thermoelestic strain

\section{Introduction}

The martensitic transformation in $\mathrm{Ni}-\mathrm{Ti}$ shape memory alloy (SMA) films is of technological interest for microactuators in micro-electro-mechanical systems (MEMS) and has thus received wide attention. ${ }^{1,2)}$ SMA films in MEMS devices can be scaled from millimeter to submicron dimensions. A conventional system, for example, is a thin film on a substrate. Such a thin film normally has an extremely small thickness compared to its lateral dimension, and thus possesses two-dimensional (2-D) characteristics. Highly developed micro fabrication technologies allow components of different dimensionality to be integrated. In order to successfully incorporate SMA films into MEMS devices, dimensional effects on SMA films need to be understood. The field of SMA film integra-tions may be divided into three sub-fields as shown in Fig. 1.

a) One-dimensional (1-D) films on (1-D) substrates, Fig. $1(\mathrm{a})$

b) Two-dimensional (2-D) films on (2-D) substrates, Fig. 1(b),

c) One-dimensional (1-D) films on (2-D) substrates, Fig. $1(\mathrm{c})$.

This paper deals with the third category. It is shown that both the thermoelastic as well as the SMA transformation induced

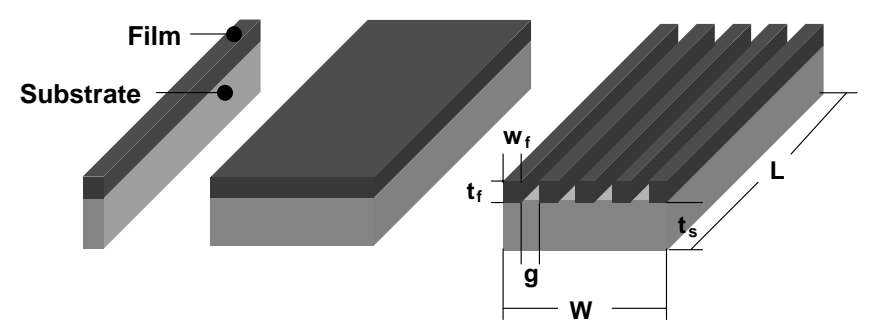
(a) 1-D/1-D
(b) 2-D/2-D
(c) 1-D/2-D

Fig. 1 Three types of composites with different dimensionality: (a) 1-D film/1-D substrate, (b) 2-D film/2-D substrate and (c) 1-D film/2-D substrate. $W$ : substrate width, $L$ : substrate length, $w_{\mathrm{f}}$ : film width, $w_{\mathrm{g}}$ : gap width, $t_{\mathrm{f}}$ : film thickness, $t_{\mathrm{s}}$ : substrate thickness.

\footnotetext{
*Now at Panasonic Boston Laboratory, Cambridge, MA.
}

strain depend on the dimensionality of the film.

\section{Experimental Techniques}

Nominally $\mathrm{Ni}_{50} \mathrm{Ti}_{50}$ targets were used to sputter deposit films on bulk micro-machined silicon (100) cantilever substrates with a $\mathrm{Si}_{3} \mathrm{~N}_{4}$ diffusion barrier layer $(100 \mathrm{~nm})$, using a dc magnetron sputtering system. The Si cantilevers ( $L=20 \mathrm{~mm}, W=4 \mathrm{~mm}, t_{\mathrm{s}}=0.07 \mathrm{~mm}$, see Fig. 1) were fabricated using conventional bulk micromachining techniques. ${ }^{3)}$ The length and width of the cantilever were aligned along $\langle 110\rangle$ directions of the Si wafer. A Shipley 1813 photoresist was spun on the surface of the Si cantilevers and patterned into periodic strips ranging from 2.5 to $1000 \mu \mathrm{m}$ in width. ${ }^{4}$ The photo masks were configured into periodic strips separated by gaps of the same width as that of the strips though final film/gap configurations deviated somewhat from the mask dimension, see Table 1. The deposition parameters were: $1.3 \cdot 10^{-4} \mathrm{~Pa}$ base vacuum, $1.3 \mathrm{~Pa}$ Ar gas pressure, $100 \mathrm{~W}$ power, $30 \mathrm{~min}$ deposition time. During the deposition the substrate was maintained at room temperature. The deposition rate was approximately $0.5 \mathrm{~nm} \mathrm{~s}^{-1}$ and the total thickness, as measured with a Sloan Dektak scanner, approximately $0.9 \mu \mathrm{m}$ for each of the so deposited films. Films of the same thickness were deposited simultaneously onto the photoresist patterned Si cantilevers so that their properties could be directly compared to each other. A lift-off process with acetone revealed the striped films. The stripes were crystallized and stress relieved by annealing for 2 hour at $500^{\circ} \mathrm{C}$ in high vac-

Table 1 Dimensions of the crystalline NiTi strips on Si cantilevers. All films were of equal thickness, $t_{\mathrm{f}}=0.9 \mu \mathrm{m}$.

\begin{tabular}{ccccc}
\hline $\begin{array}{c}\text { Sample } \\
\text { Nr. }\end{array}$ & $\begin{array}{c}\text { Width of } \\
\text { strip } \\
w_{\mathrm{f}} / \mu \mathrm{m}\end{array}$ & $\begin{array}{c}\text { Distance } \\
\text { between strips } \\
g / \mu \mathrm{m}\end{array}$ & $\begin{array}{c}\text { Aspect ratio } \\
t_{\mathrm{f}} / w_{\mathrm{f}}\end{array}$ & $\begin{array}{c}\text { Actual areal } \\
\text { density } \\
w_{\mathrm{f}} /\left(w_{\mathrm{f}}+g\right)[\%]\end{array}$ \\
\hline 1 & 2.9 & 2.1 & 0.31 & 58.0 \\
2 & 5.4 & 4.6 & 0.16 & 54.0 \\
3 & 54 & 46 & 0.016 & 54.0 \\
4 & 1000 & 1000 & 0.0009 & 50.0 \\
\hline
\end{tabular}



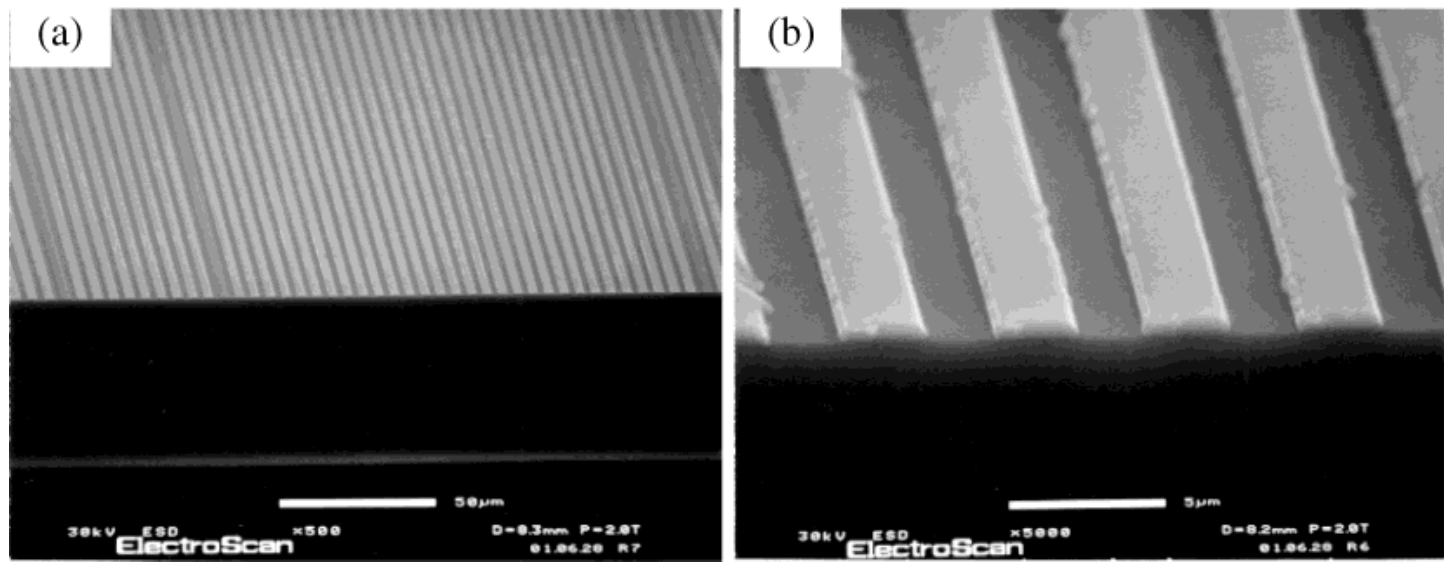

Fig. 2 SEM images of patterned NiTi films on a Si cantilever substrate: (a) $\times 500$ and (b) $\times 5000$.

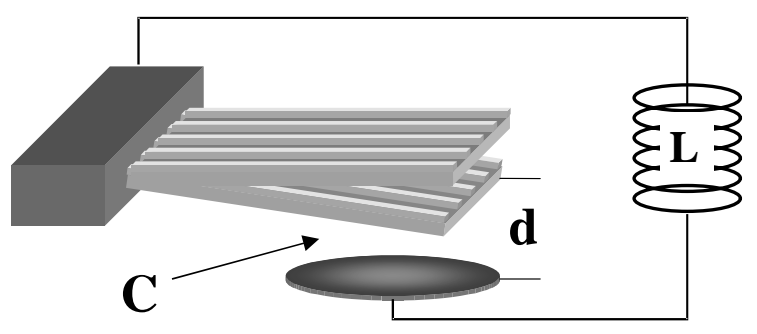

Fig. 3 Principle of measurement, the cantilever deflection is determined from the change of the resonance frequency $\omega_{\mathrm{r}}=\frac{1}{\sqrt{L C}}$.

uum. Figure 2 shows SEM pictures of micro patterned NiTi films on a Si cantilever. The final dimensions of the patterned films are shown in Fig. 1(c) in conjunction with Table 1. The SEM examination affirmed that all films had the same thickness. The deflection of the NiTi/Si composite cantilevers due to thermo-mechanical stresses and the occurrence of the martensitic transformation in the SMA film were measured as a function of temperature by using a capacitive technique. ${ }^{5)}$ The diameter of the capacitance equaled approximately the sample width. The measurement principle is shown in Fig. 3. All measurements were performed using identical heating and cooling rates in a helium gas atmosphere.

\section{Results and Discussion}

Figure 4 displays the main result of the study, the temperature dependence of the deflection of the four differently patterned $\mathrm{NiTi} / \mathrm{Si}$ composite cantilevers. In order to facilitate a direct comparison of the data the deflection $\delta$ displayed in Fig. 4 has been normalized to the same total NiTi film volume, i.e. $\delta=d \frac{w_{\mathrm{f}}+g}{w_{\mathrm{f}}}$. Here, the quantity $d$ is the experimentally observed cantilever deflection indicated in Fig. 3. The patterns differed in the aspect ratios of the NiTi strips as given in Table 1.

The data in Fig. 4 show that the deflection of the NiTi/Si composite cantilever depends on the aspect ratio $t_{\mathrm{s}} / w$ in the high, $T>A_{\mathrm{f}}$ or $M_{\mathrm{s}}$ (austenite phase), intermediate $A_{\mathrm{s}}<T<$ $A_{\mathrm{f}}$ or $M_{\mathrm{f}}<T<M_{\mathrm{s}}$ (austenite plus martensite phases), and low, $T<A_{\mathrm{s}}$ or $M_{\mathrm{f}}$ (martensite phase), temperature regimes. Quantitatively, the deflection is measured by the slope $\mathrm{d} \delta / \mathrm{d} T$

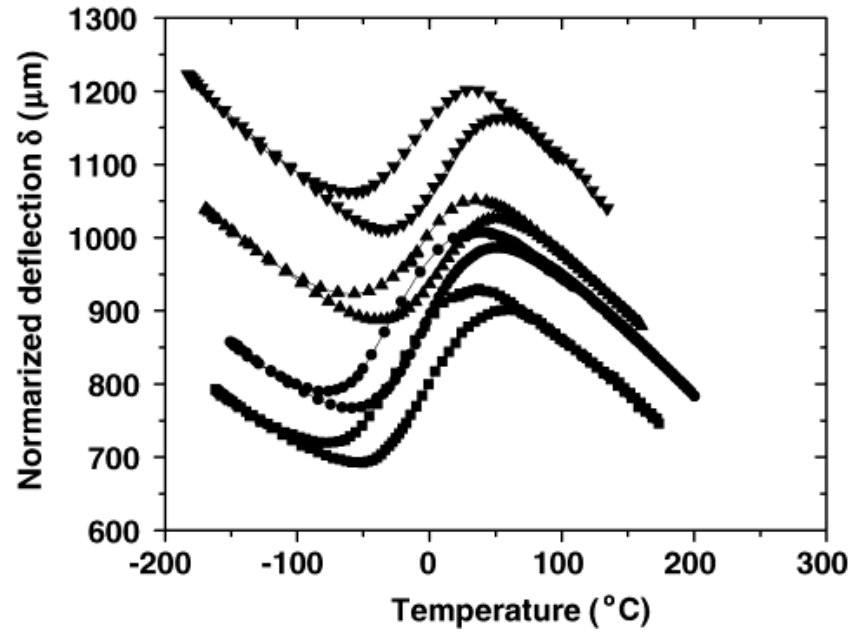

Fig. 4 Deflection of four differently patterned NiTi/Si bimorph cantilevers as a function of temperature. All bimorphs were processed simultaneously and identically. $\square \square$ (full squares)-sample 1 aspect ratio $=0.31$, - (full circles)-sample 2 as pect ratio $=0.16, \boldsymbol{\Delta} \boldsymbol{\Delta} \boldsymbol{\Delta}$ (tip up full triangles)-sample 3 aspect ratio $=0.016, \boldsymbol{\nabla} \nabla$ (tip down full triangles)-sample 4 aspect ratio $=0.0009$. See Table 1 for sample dimensions. The density of data points has been reduced by a factor of five.

of the linear portion of the graph $\delta=f(T)$ in the respective temperature regime. A larger aspect ratio results in a smaller thermoelastic deflection with a negative slope $\mathrm{d} \delta / \mathrm{d} T<0$ in the high and low temperature regimes. In the intermediate temperature regime the transformation induced deflection has a positive slope $\mathrm{d} \delta / \mathrm{d} T>0$.

The thermoelastic deflection occurs due to a mismatch stress in the NiTi/Si composite. A simple stress analysis of the 1-D film/2-D substrate composite is depicted in Fig. 5. The NiTi thin film has a higher coefficient of thermal expansion than that of the Si substrate, and thus shrinks faster on cooling from the annealing temperature (see Fig. 5(a)). The NiTi film needs to be stretched by a biaxial tensile stress $\sigma_{\mathrm{w}}^{\mathrm{f}}=\sigma_{\mathrm{L}}^{\mathrm{f}}$ to be fit onto the substrate, see Fig. 5(b), Contrarily, the Si substrate is subjected to a biaxial compressive stress $-\sigma_{\mathrm{w}}^{\mathrm{s}}=-\sigma_{\mathrm{L}}^{\mathrm{s}}$ in order to match the interface dimensions, see Fig. 5(b). The film is attached to the substrate to form the NiTi/Si composite structure. Since the geometry of the striped NiTi film is one dimensional, the stress in the $\mathrm{W}$-direction is relaxed as 


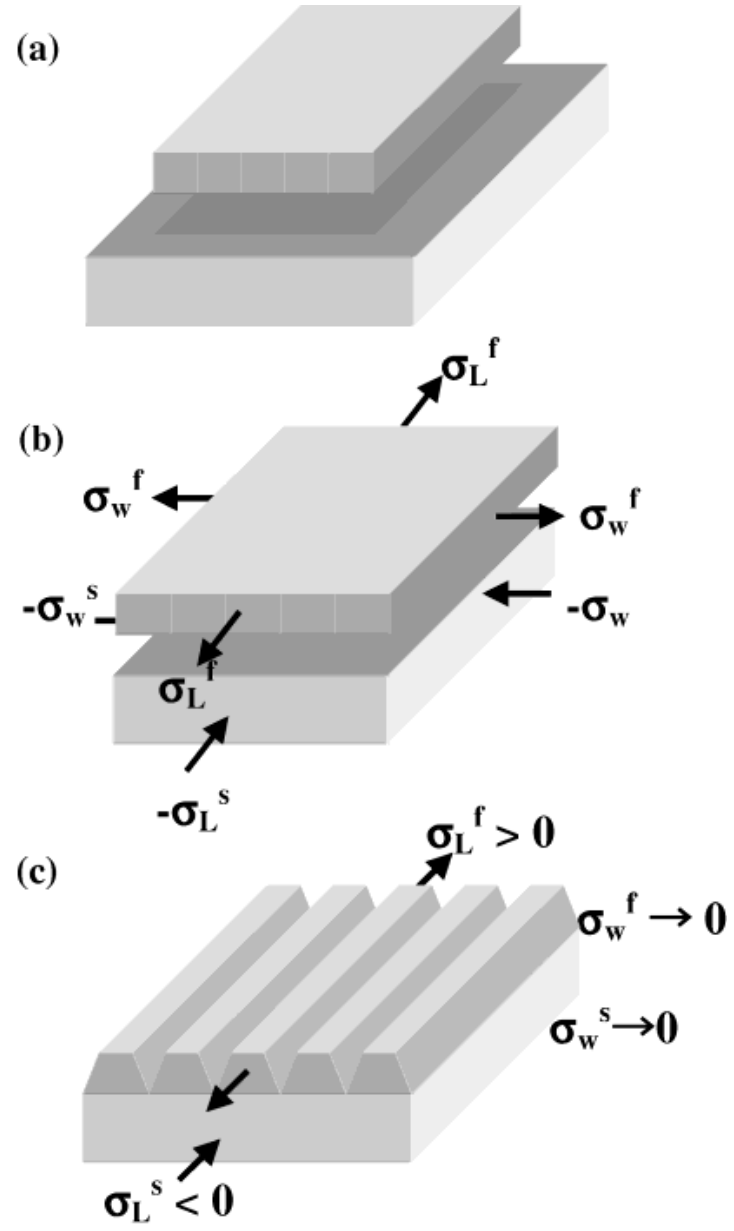

Fig. 5 Simple stress analysis of a 1-D/2-D composite: (a) unstressed compo-nents, (b) components stressed biaxially, (c) patterned film and substrate stressed uniaxially.

sketched schematically in Fig. 5(c). ${ }^{6,7)}$ It is noted that the amount of relaxation depends on the aspect ratio of the NiTi thin film. ${ }^{8)}$ Therefore, the stress state of the 1-D film/2-D substrate composite becomes uniaxial while the stress state of the conventional 2-D film/2-D substrate composite remains biaxial.

The specific thermoelastic deflection $\delta^{\prime}=2 \delta / L^{2}$, where $L$ is the sample length, of the composite cantilever is analyzed using Stoney's equation. ${ }^{9)}$ For biaxially stressed film this equation can be used as is

$$
\delta_{\mathrm{bi}}^{\prime} \approx \frac{6 t_{\mathrm{f}}}{t_{\mathrm{s}}^{2}} \frac{E_{\mathrm{f}}\left(1-v_{\mathrm{s}}\right)}{E_{\mathrm{s}}\left(1-v_{\mathrm{f}}\right)} \Delta \varepsilon .
$$

However, for the present uniaxially stressed NiTi stripes and Si cantilevers it must be modified to

$$
\delta_{\text {uni }}^{\prime} \approx \frac{6 t_{\mathrm{f}}}{t_{\mathrm{s}}^{2}} \frac{E_{\mathrm{f}}}{E_{\mathrm{s}}} \Delta \varepsilon
$$

In these equations the quantities $t_{\mathrm{f}}$ and $t_{\mathrm{s}}$ represent the thickness of film and substrate, and $E_{\mathrm{f}}$ and $E_{\mathrm{s}}$ are their Young's moduli. The constants $v_{\mathrm{f}}$ and $v_{\mathrm{s}}$ denote Poisson's ratios of the film and substrate, respectively. Their numbers are tabulated in Table 2 . The quantity $\Delta \varepsilon$ denotes the strain in the composite. The right hand side of Stoney's equations is divided into three terms as shown in Table 4 to highlight the difference of the deflections between uniaxial and biaxial stress states:
Table 2 Mechanical properties of NiTi and Si single crystal. ${ }^{10,11)}$

\begin{tabular}{ccc}
\hline & $\mathrm{NiTi}$ & $\mathrm{Si}(100)\langle 110\rangle$ \\
\hline$E(\mathrm{GPa})$ & $83(\mathrm{~A})$ & 170 \\
$v$ & $28-41(\mathrm{M})$ & 0.048 \\
\hline
\end{tabular}

geometrical, modulus, and strain terms.

1. The geometrical terms are constant and equal for both equations.

2. The modulus terms play a role in the deflection of the composite in all temperature ranges. If Poisson's ratios of film and substrate are equal, for example, the deflections for uniaxial and biaxial stress states do not differ (e.g. polycrystalline substrate and polycrystalline film). However, the deflections will be different if Poisson's ratios of film and substrate are not equal as is the case here. ${ }^{12}$ ) The difference of the deflections between uniaxial and biaxial stress states results in the ratio $\frac{1-v_{\mathrm{s}}}{1-v_{\mathrm{f}}}$ as originally derived. From this ratio and the values of Poisson's ratios given in Table 2 it follows that the thermoelastic displacement of the 1-D NiTi/2-D Si composite is expected to be lower than that of the 2-D NiTi/2-D Si in the single-phase temperature regions, $T>A_{\mathrm{f}}, M_{\mathrm{s}}$, and $T<A_{\mathrm{s}}, M_{\mathrm{f}}$. The stress state thus explains that high aspect ratio 1-D films show smaller magnitudes and slopes of the thermoelastic deflection.

3. The strain terms $\Delta \varepsilon$ are given by the difference of the coefficients of thermal expansion $\Delta \alpha \Delta T$ or by the martensitic transformation strain, $\varepsilon_{\mathrm{M}}$. The coefficients of thermal expansion are only minimally, if at all, effected by the stress states. However, the strain resulting from the martensitic transformation differs for the uniaxial and biaxial stress states in the intermediate temperature regime, $M_{\mathrm{f}}, A_{\mathrm{s}},<T<M_{\mathrm{s}}, A_{\mathrm{f}}$.

The magnitude of the strain $\varepsilon_{\mathrm{M}}$ is determined by the crystallinity of the film, i.e. single versus polycrystalline, its texture, the nature of the phase transformation as well as the state of stress prevailing during the transformation. The cubic-tetragonal transformation represents an extreme example of the influence of the state of stress. Consider the cubictetragonal transformation of single crystalline 1-D and 2-D films on a Si substrate as shown in Fig. 6. For a biaxial state of stress, the microstructure consists of equal parts of domains of different eigenstrains ${ }^{13)}$

$$
\begin{aligned}
& \frac{1}{2}\left(\begin{array}{ccc}
\varepsilon_{0} & 0 & 0 \\
0 & 0 & 0 \\
0 & 0 & -\varepsilon_{0}
\end{array}\right)+\frac{1}{2}\left(\begin{array}{ccc}
0 & 0 & 0 \\
0 & \varepsilon_{0} & 0 \\
0 & 0 & -\varepsilon_{0}
\end{array}\right) \\
& =\left(\begin{array}{ccc}
\frac{1}{2} \varepsilon_{0} & 0 & 0 \\
0 & \frac{1}{2} \varepsilon_{0} & 0 \\
0 & 0 & -\varepsilon_{0}
\end{array}\right)
\end{aligned}
$$

whereas for a uniaxial state of stress, $\sigma_{\mathrm{f}, \mathrm{L}} \gg \sigma_{\mathrm{f}, \mathrm{w}}$, domains 

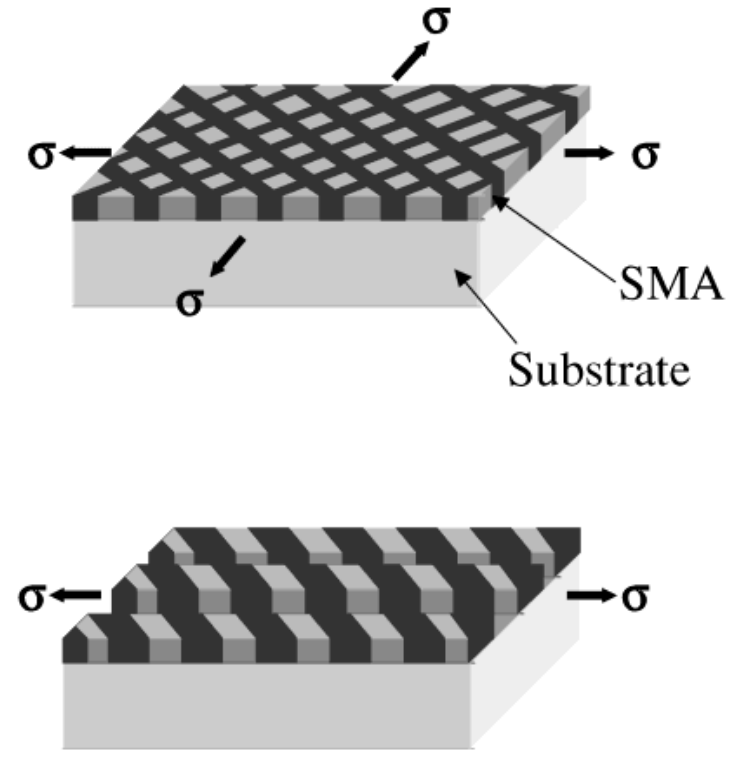

Fig. 6 Conceptual martensitic microstructure of biaxially (left) and uniaxially (right) stressed SMA films in the two phase temperature region. The light and dark sections represent martensite and austenite, respectively.

Table 3 Experimentally determined slopes $\mathrm{d} \delta / \mathrm{d} T$.

\begin{tabular}{cccc}
\hline Aspect ratio $t_{\mathrm{f}} / w_{\mathrm{f}}$ & $T<A_{\mathrm{s}}$ & $A_{\mathrm{s}}<T<A_{\mathrm{f}}$ & $A_{\mathrm{f}}<T$ \\
\hline 0.31 & -1.27 & 1.95 & -1.42 \\
0.16 & -1.29 & 2.01 & -1.39 \\
0.016 & -1.37 & 1.55 & -1.53 \\
0.0009 & -1.49 & 1.56 & -1.68 \\
\hline
\end{tabular}

with the eigenstrain

$$
\begin{aligned}
& \frac{1}{2}\left(\begin{array}{ccc}
0 & 0 & 0 \\
0 & \varepsilon_{0} & 0 \\
0 & 0 & -\varepsilon_{0}
\end{array}\right)+\frac{1}{2}\left(\begin{array}{ccc}
0 & 0 & 0 \\
0 & \varepsilon_{0} & 0 \\
0 & 0 & -\varepsilon_{0}
\end{array}\right) \\
& =\left(\begin{array}{ccc}
0 & 0 & 0 \\
0 & \varepsilon_{0} & 0 \\
0 & 0 & -\varepsilon_{0}
\end{array}\right)
\end{aligned}
$$

dominate. It follows that for $\sigma_{\mathrm{f} . \mathrm{L}}=\sigma_{\mathrm{f}, \mathrm{w}}, \varepsilon_{\mathrm{M}}=\frac{\varepsilon_{0}}{\beta}, \beta=2$, whereas for $\sigma_{\mathrm{f} . \mathrm{L}} \gg \sigma_{\mathrm{f}, \mathrm{w}}, \varepsilon_{\mathrm{M}}=\varepsilon_{0}$. Note that the $z$-component of the eigenstrain is immaterial to the problem. ${ }^{6}$ )

The experimental values of the slopes $\mathrm{d} \delta / \mathrm{d} T$ on heating are listed in Table 3. They were determined from a linear least square fit to the data that corresponded to the maximum of the correlation coefficient for the temperature regimes, $T>A_{\mathrm{f}}$ and $T<A_{\mathrm{s}}$. The slope in the transformation region, $A_{\mathrm{s}}<$ $T<A_{\mathrm{f}}$, was determined from the change of the deflection and temperature between $A_{\mathrm{s}}$ and $A_{\mathrm{f}}, \frac{\delta_{A_{\mathrm{f}}}-\delta_{A_{\mathrm{s}}}}{T_{A_{\mathrm{f}}}-T_{A_{\mathrm{s}}}}$.

The experimental quantity amenable to analysis is the ratio of the slopes $P=\frac{\left(\mathrm{d} \delta^{\prime} / \mathrm{d} T\right)_{\mathrm{H}}}{\left(\mathrm{d} \delta^{\prime} / \mathrm{d} T\right)_{\mathrm{L}}}$ of the high and low aspect ratio composites. The steps to evaluate this ratio of slopes are presented in Table 4. The modulus $E_{\mathrm{f}}^{\prime}$ as well as $v_{\mathrm{f}}^{\prime}$ in the two phase regime are complex quantities as they reflect not only elastic deformations but also those mediated by the motion of twin boundaries. The following analysis treats them as independent of temperature and dimensionality.
In the high temperature regime $T>A_{\mathrm{f}}$ the strain is determined by the difference of the coefficients of thermal expansion of the high temperature B2 phase of NiTi and of silicon, $\Delta \alpha$. In the temperature range $A_{\mathrm{s}}<T<A_{\mathrm{f}}$ it is determined by the martensitic transformation strain. In preparing the table the thermoelastic strain was neglected, as it is smaller than the transformation strain. The temperature dependent transformation strain $\varepsilon_{\mathrm{M}} \cdot \gamma(T)$ reflects the state of stress at which the martensite is formed. It is composed of the transformation strain $\varepsilon_{\mathrm{M}}$ and a parameter $\gamma(T)$ representing the temperature dependent microstructure of the transforming film. In the following it is assumed that $\gamma(T)$ does not change significantly with the dimensionality of the film.

The steps shown in Table 4 permit an approximate evaluation of the ratios of the slopes $\frac{\mathrm{d} \delta}{\mathrm{d} T}$ for high and low aspect ratios of the film, $P$, in the three temperature regimes $T>A_{\mathrm{f}}$ (austenite), $A_{\mathrm{s}}<T<A_{\mathrm{f}}$ (transformation) and $T<A_{\mathrm{s}}$ (martensite). According to Table 4 the slopes $\left(\frac{\mathrm{d} \delta}{\mathrm{d} T}\right)_{\mathrm{H}}$ should approximately be 0.7 times lower than $\left(\frac{\mathrm{d} \delta}{\mathrm{d} T}\right)_{\mathrm{L}}$ in the single phase regions $T>A_{\mathrm{f}}$ and $T<A_{\mathrm{s}}$. The ratio of slopes in the transformation region can vary from 0.7 to 1.4 depending on the evolution and nature of the martensite microstructure: for a single crystalline cubic $\rightarrow$ tetragonal transformation, as can be seen by comparing eqs. (3) and (4), $\beta=2$. Hence $P_{\text {est }}=1.4$ as an extreme case for a cubic-tetragonal transformation. This estimate compares well with the experimental data, $P_{\exp }$, that can be obtained from Table 2 and are included in Table 3, i.e. $P_{\text {est }} \approx P_{\text {exp }}$. The dimensional aspects of the stress relief characteristics of monoclinic and tetragonal martensites thus closely resemble each other. This can be understood by considering the monoclinic martensite as a distorted tetragonal structure. It might be noted that this result is independent of or weakly dependent on the film modulus $E_{\mathrm{f}}^{\prime}$. The shift of the transformation temperatures with increasing aspect ratio evidenced in Fig. 4 results from their known stress dependence.

In summary it can be stated that SMA/Si bimorph cantilevers with a SMA film patterned into strips show a smaller thermoelastic and a larger transformational deflection than the same bimorphs with unpatterned, planar SMA films. The difference is the result of the stress states of the composite that are determined by the dimensionality of the film, as well as the effectiveness of the martensite variants that are responsible for the deflection in the two phase regime. The magnitude of Poisson's ratio of silicon contributes significantly to the observed effects.

\section{Acknowledgements}

This work is supported by the Office of Naval Research, contracts No. N000149910837 and N000140010849 and MURI N000140110761. It also benefited from the support of the National Science Foundation, grant DMR0095166.

\section{REFERENCES}

1) S. Miyazaki and A. Ishida: Mater. Sci. and Eng. A273-275 (1999) 106133.

2) T. Matsunaga, S. Kajiwara, K. Ogawa, T. Kikuchi and S. Miyazaki: Mater. Sci. Eng. A273-275 (1999) 745-748. 
Table 4 Evaluation of slopes of deflection and comparison of ratios.

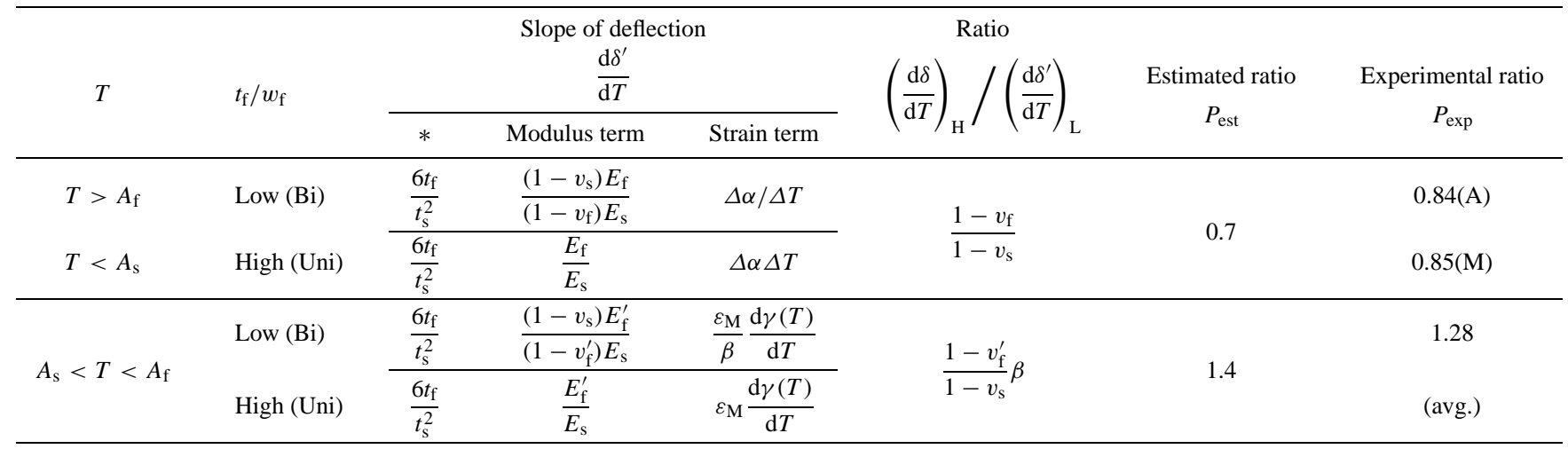

*The experiment was guided such that this geometrical term is a constant.

3) J. Morillo, Q. Su, D. Novotny and M. Wuttig: Rev. Sci. Instrum. 69 (1998) 3908-3914.

4) M. Madou: Fundamentals of microfabrication, (CRC Press LLC, Florida, 1997).

5) Manfred Wuttig and C. M. Su: Proc. Symp. on Damping in Multiphase Inorganic Materials, R. B. Bhagat, Ed. (AMS, Metals Park, OH, 1993) pp. 159-165.

6) K. P. A. H. Harker, S. C. Jain, A. Atkinson and R. Bullough: Philos. Mag. A71 (1995) 871-881.

7) T. Hanabusa: Mat. Sci. Res. Intr. 5 (1999) 63-73.
8) A. L. Roytbutd, S. P. Alpay, V. Nagarajan, C. S. Gampule, S. Aggarwal, E. D. Williams and R. Ramesh: Phys. Rev. Lett. 85 (2000) 190-193.

9) S. Timoshenko: J. Opt. Soc. 11 (1925) 233-234.

10) J. J. Wortman and R. A. Evans: J. Appl. Phys. 36 (1965) 153.

11) http://www.sma-inc.com/NiTiProperties.html, Shape Memory Applications Inc.

12) M. Finot and S. Suresh: J. Mech. Phys. Solids 44 (1996) 683-721.

13) A. L. Royburd, T. Kim, Q. Su and M. Wuttig: Acta Mater. 46 (1998) 5095-5107. 